\title{
A combination theorem for strong relative hyperbolicity
}

\author{
MAHAN MJ \\ LAWRENCE REEVES
}

\begin{abstract}
We prove a combination theorem for trees of (strongly) relatively hyperbolic spaces and finite graphs of (strongly) relatively hyperbolic groups. This gives a geometric extension of Bestvina and Feighn's Combination Theorem for hyperbolic groups and answers a question of Swarup. We also prove a converse to the main Combination Theorem.
\end{abstract}

20F67; 57M50

\section{Introduction}

In [4], Bestvina and Feighn proved a combination theorem for hyperbolic groups. Motivated by this, Swarup asked the analogous question [3] for relatively hyperbolic groups. Dahmani [9] and Alibegović [1] have proven combination theorems motivated by applications to convergence groups and limit groups (cf Sela [28]).

In this paper, we prove a geometric combination theorem (as opposed to a dynamical one) for trees of (strong) relatively hyperbolic metric spaces. We use Bestvina and Feighn's Combination Theorem [4] directly in deducing the relevant combination theorem. The conditions we impose are quite different from those of [9] and [1]. Our main Theorems 4.5 and 4.7 are stated below:

Strong Combination Theorem and converse: Theorem 4.5 and Theorem 4.7 Let $X$ be a tree ( $T$ ) of strongly relatively hyperbolic spaces satisfying:

(1) the q(uasi)-i(sometrically)-embedded condition

(2) the strictly type-preserving condition

(3) the qi-preserving electrocution condition

(4) the induced tree of coned-off spaces satisfies the hallways flare condition

(5) the cone-bounded hallways strictly flare condition. 
Then $X$ is strongly hyperbolic relative to the family $\mathcal{C}$ of maximal cone-subtrees of horosphere-like spaces.

Conversely, if $X$ be a tree ( $T$ ) of strongly relatively hyperbolic spaces satisfying conditions (1)-(3) such that $X$ is strongly hyperbolic relative to the family $\mathcal{C}$ of maximal cone-subtrees of horosphere-like spaces, then the tree of spaces satisfies conditions (4)-(5).

Of the conditions given in the above Theorem, Condition (1) is taken directly from Bestvina and Feighn [4]. Condition (2) roughly says that the preimage of a horospherelike subset (thought of as parabolic) in a vertex space (under the edge-space to vertexspace map) is either empty or a horosphere-like subset in the corresponding edgespace. This condition may be likened to the restriction to strictly type-preserving maps in the theory of Kleinian groups. Condition (2) ensures an induced tree of electrocuted spaces. Condition (3) says that the induced tree of spaces also satisfies the qi-embedded condition. Condition (4) is again taken directly from [4]. Condition (5) is the one essential new condition. It says roughly that a pair of geodesics whose vertices consist only of cone-points cannot lie close to each other for long. The notion of fully quasiconvex subgroups introduced by Dahmani [9] is related to Condition (3), the qi-preserving electrocution condition.

Note In this paper we adopt the convention that the are horosphere-like subsets are coarsely proper, ie no finite neighborhood of a horosphere-like subset is the whole space (cf Behrstock, Drutu and Mosher [2] which follows a similar convention). This excludes the trivial case that $X$ is strongly hyperbolic relative to itself, or a net in $X$. This assumption translates into the context of groups. Hence we assume that if a group $G$ is strongly hyperbolic relative to a collection of subgroups, then no subgroup $H$ in the collection is of finite index in $G$.

As an immediate consequence of Theorem 4.5, we have:

Strong Combination Theorem for graphs of groups: Theorem 4.6 Let $G$ be a finite graph $(\Gamma)$ of strongly relatively hyperbolic groups satisfying:

(1) the qi-embedded condition

(2) the strictly type-preserving condition

(3) the qi-preserving electrocution condition

(4) the induced tree of coned-off spaces satisfies the hallways flare condition

(5) the cone-bounded hallways strictly flare condition. 
Then $G$ is strongly hyperbolic relative to the family $\mathcal{C}$ of maximal parabolic subgroups.

All these conditions are satisfied in the classical case of a 3-manifold fibering over the circle with fiber a punctured surface. The one condition that needs checking is the hallways flare condition for the induced tree (in fact line) of coned-off spaces. This fact is due to Bowditch [7, Section 6]. The verification involves using the associated singular structure coming from stable and unstable foliations. We shall give a slightly modified version, using an idea of Mosher [26] to show this. (See Section 4.3). In fact we prove the stronger Theorem:

Theorem 4.9 Let $\Phi_{1} \cdots \Phi_{m}$ be $m$ pseudo-Anosov diffeomorphisms of $\Sigma$ with different sets of stable and unstable foliations. Let $H=\pi_{1}(\Sigma)$. Then there is an $n \geq 1$ such that the diffeomorphisms $\Phi_{1}^{n}, \cdots \Phi_{m}^{n}$ generate a free group $F$ and the group $G$ given by the exact sequence

$$
1 \rightarrow H \rightarrow G \rightarrow F \rightarrow 1
$$

is (strongly) hyperbolic relative to the maximal parabolic subgroups of the form $\mathcal{Z} \times F$.

We remark here that in Dahmani's combination theorem [9] an essential condition is acylindricity. Again, in Alibegović's combination theorem [1], an essential assumption is the compact intersection property. Both acylindricity and the compact intersection property prevent infinite (or even arbitrarily long chains of parabolics from occurring). To us, this seemed a bit unsatisfactory, as the original motivation for the BestvinaFeighn result came from Thurston's monster theorem (see Kapovich [16]), and we wanted a generalization of the Bestvina-Feighn theorem that would cover the case of hyperbolic 3-manifolds with parabolics, particularly hyperbolic 3-manifolds of finite volume fibering over the circle. The hypotheses in the present paper do allow for infinite chains of parabolics and covers the above case. Our emphasis here is geometric and so the main theorem is stated in terms of spaces rather than groups.

Acknowledgements The authors are grateful to G A Swarup, who was instrumental in bringing about this collaborative effort. The work was completed during a visit of the second author to RKM Vivekananda University in February 2006. We are also grateful to the referee for helpful comments and corrections. ${ }^{1}$

\footnotetext{
${ }^{1}$ After the submission of this paper, we learnt of the paper [11] by Gautero, which gives a different proof of a result equivalent to Theorem 4.6. A couple of points of difference between our work and [11]: We use Bestvina-Feighn's result [4] directly, whereas an alternate proof of the Combination Theorem of [4] is provided in [11]. However, in this paper, we provide in addition, a converse (Theorem 4.7) to the main Combination Theorem.
} 


\section{Relative hyperbolicity}

In this section, we shall first recall certain notions of relative hyperbolicity due to Farb [10] and Gromov [14].

\subsection{Electric geometry}

Let $X$ be a path metric space. A collection of closed subsets $\mathcal{H}=\left\{H_{\alpha}\right\}$ of $X$ will be said to be uniformly separated if there exists $\epsilon>0$ such that $d\left(H_{1}, H_{2}\right) \geq \epsilon$ for all distinct $H_{1}, H_{2} \in \mathcal{H}$.

Definition 2.1 (Farb [10]) The electric space (or coned-off space) $\widehat{X}$ corresponding to the pair $(X, \mathcal{H})$ is a metric space which consists of $X$ and a collection of vertices $v_{\alpha}$ (one for each $H_{\alpha} \in \mathcal{H}$ ) such that each point of $H_{\alpha}$ is joined to (coned off at) $v_{\alpha}$ by an edge of length $1 / 2$. The sets $H_{\alpha}$ shall be referred to as horosphere-like sets.

A geodesic (resp. quasigeodesic) in $\widehat{X}$ will be referred to as an electric geodesic (resp. quasigeodesic).

Definition 2.2 A path $\gamma: I \rightarrow X$ in a path metric space $X$ is an ambient $K$-quasigeodesic if we have

$$
L(\beta) \leq K L(A)+K
$$

for any subsegment $\beta=\gamma \mid[a, b]$ and any rectifiable path $A:[a, b] \rightarrow Y$ with the same endpoints. (Here $L$ denotes length of path.)

$N_{R}(Z)$ will denote the $R$-neighborhood about the subset $Z$ in $X . N_{R}^{e}(Z)$ will denote the $R$-neighborhood about the subset $Z$ in the electric metric.

Much of what Farb proved in [10] goes through under considerably weaker assumptions than those of [10]. In [10] the theorems were proven in the particular context of a pair $(X, \mathcal{H})$, where $X$ is a Hadamard space of pinched negative curvature with the interiors of a family of horoballs $\mathcal{H}$ removed. Then $\mathcal{H}$ can be regarded as a collection of horospheres in $X$ separated by a minimum distance from each other. In this situation, $X$ is not a hyperbolic metric space itself, but is hyperbolic relative to a collection of separated horospheres. Alternately let $H_{h}$ be the horoball corresponding to the horosphere $H \in \mathcal{H}$. Let $X_{h}=X \bigcup_{H \in \mathcal{H}} H_{h}$ be the entire Hadamard manifold of pinched negative curvature. Then the coned-off space $\widehat{X}$ obtained by coning off the horospheres of $X$ is essentially equivalent to coned-off space $\widehat{X}_{h}$ obtained by coning off the horoballs of $X_{h}$. 
We consider therefore a hyperbolic metric space $X$ and a collection $\mathcal{H}$ of (uniformly) $C$-quasiconvex uniformly separated subsets, ie there exists $D>0$ such that for $H_{1}, H_{2} \in \mathcal{H}, d_{X}\left(H_{1}, H_{2}\right) \geq D$. In this situation $X$ is weakly hyperbolic relative to the collection $\mathcal{H}$ in the sense that the coned-off space $\widehat{X}$ is hyperbolic. The result in this form is due to Klarreich [17]. However, the property of Bounded Horosphere Penetration (BHP) or Bounded Coset Penetration (BCP) used by Farb [10] was not abstracted out in Klarreich's proof as it was not necessary. What is essential for BCP (or BHP) to go through has been abstracted out by Bowditch $[7 ; 6]$ in the case that the collection $\mathcal{H}$ is a collection of geodesics or horocycles in a Farey graph. (See also Bumagin [8].) But though these things are available at the level of folklore, an explicit statement seems to be lacking.

The crucial condition can be isolated as per the following definition [23]:

Definition 2.3 A collection $\mathcal{H}$ of uniformly $C$-quasiconvex sets in a $\delta$-hyperbolic metric space $X$ is said to be mutually $D$-cobounded if for all $H_{i}, H_{j} \in \mathcal{H}, \pi_{i}\left(H_{j}\right)$ has diameter less than $D$, where $\pi_{i}$ denotes a nearest point projection of $X$ onto $H_{i}$. A collection is mutually cobounded if it is mutually $D$-cobounded for some $D$.

Mutual coboundedness was proven for horoballs by Farb in Lemma 4.7 of [10] and by Bowditch in stating that the projection of the link of a vertex onto another [6] has bounded diameter in the link. However, the comparability of intersection patterns in this context needs to be stated a bit more carefully. We give the general version of Farb's theorem below and refer to [10] and Klarreich [17] for proofs.

Lemma 2.4 (See Lemma 4.5 and Proposition 4.6 of Farb [10] and Mj [23].) Given $\delta, C, D$ there exists $\Delta$ such that if $X$ is a $\delta$-hyperbolic metric space with a collection $\mathcal{H}$ of $C$-quasiconvex $D$-separated sets. then we have the following:

(1) Electric quasigeodesics electrically track hyperbolic geodesics: Given $P>0$, there exists $K>0$ with the property that if $\beta$ is any electric $P$-quasigeodesic from $x$ to $y$, and $\gamma$ is the hyperbolic geodesic from $x$ to $y$, then $\beta \subset N_{K}^{e}(\gamma)$.

(2) $\gamma$ lies in a hyperbolic $K$-neighborhood of $N_{0}(\beta)$, where $N_{0}(\beta)$ denotes the zero neighborhood of $\beta$ in the electric metric.

(3) Hyperbolicity: The electric space $\widehat{X}$ is $\Delta$-hyperbolic.

Item (2) in the above Lemma is due to Klarreich [17], where the proof is given for $\beta$ an electric geodesic, but the same proof goes through for electric quasigeodesics without backtracking. 
The above Lemma motivates:

Definition $2.5[10 ; 6]$ Let $X$ be a geodesic metric space and $\mathcal{H}$ be a collection of mutually disjoint uniformly separated subsets. Then $X$ is said to be weakly hyperbolic relative to the collection $\mathcal{H}$, if the electric space $\widehat{X}$ is hyperbolic.

We shall need to give a general definition of geodesics and quasigeodesics without backtracking.

Definition 2.6 Given a collection $\mathcal{H}$ of $C$-quasiconvex, $D$-separated sets and a number $\epsilon$ we shall say that a geodesic (resp. quasigeodesic) $\gamma$ is a geodesic (resp. quasigeodesic) without backtracking with respect to $\epsilon$ neighborhoods if $\gamma$ does not return to $N_{\epsilon}(H)$ after leaving it, for any $H \in \mathcal{H}$. A geodesic (resp. quasigeodesic) $\gamma$ is a geodesic (resp. quasigeodesic) without backtracking if it is a geodesic (resp. quasigeodesic) without backtracking with respect to $\epsilon$ neighborhoods for some $\epsilon \geq 0$.

Note For the above Lemma, the hypothesis is that $\mathcal{H}$ consists of uniformly quasiconvex, mutually separated sets. Mutual coboundedness has not yet been used. We introduce it in the next Lemma.

Lemma 2.7 [23] Suppose $X$ is a $\delta$-hyperbolic metric space with a collection $\mathcal{H}$ of $C$-quasiconvex $K$-separated $D$-mutually cobounded subsets. There exists $\epsilon_{0}=$ $\epsilon_{0}(C, K, D, \delta)$ such that the following holds:

Let $\beta$ be an electric $P$-quasigeodesic without backtracking and $\gamma$ a hyperbolic geodesic, both joining $x, y$. Then, given $\epsilon \geq \epsilon_{0}$ there is $D=D(P, \epsilon)$ such that we have:

(1) Similar intersection patterns 1: If precisely one of $\{\beta, \gamma\}$ meets an $\epsilon$-neighborhood $N_{\epsilon}\left(H_{1}\right)$ of an electrocuted quasiconvex set $H_{1} \in \mathcal{H}$, then the length (measured in the intrinsic path-metric on $N_{\epsilon}\left(H_{1}\right)$ ) from the entry point to the exit point is at most $D$.

(2) Similar intersection patterns 2: If both $\{\beta, \gamma\}$ meet some $N_{\epsilon}\left(H_{1}\right)$ then the length (measured in the intrinsic path-metric on $N_{\epsilon}\left(H_{1}\right)$ ) from the entry point of $\beta$ to that of $\gamma$ is at most $D$; similarly for exit points.

Lemma 2.7 is essentially a paraphrasing of the $\mathrm{BCP}$ property [10] in terms of mutual coboundedness. The above Lemma motivates the following definition: 
Definition $2.8[10 ; 6]$ Let $X$ be a geodesic metric space and $\mathcal{H}$ be a collection of mutually disjoint uniformly separated subsets such that $X$ is weakly hyperbolic relative to the collection $\mathcal{H}$. If any pair of electric quasigeodesics without backtracking starting and ending at the same point have similar intersection patterns with horosphere-like sets (elements of $\mathcal{H}$ ) then quasigeodesics are said to satisfy Bounded Penetration and $X$ is said to be strongly hyperbolic relative to the collection $\mathcal{H}$.

We summarize the Lemma 2.4 and Lemma 2.7 as follows:

- If $X$ is a hyperbolic metric space and $\mathcal{H}$ a collection of uniformly quasiconvex separated subsets, then $X$ is hyperbolic relative to the collection $\mathcal{H}$.

- If $X$ is a hyperbolic metric space and $\mathcal{H}$ a collection of uniformly quasiconvex mutually cobounded separated subsets, then $X$ is hyperbolic relative to the collection $\mathcal{H}$ and satisfies Bounded Penetration, ie hyperbolic geodesics and electric quasigeodesics have similar intersection patterns in the sense of Lemma 2.7.

\subsection{Partial electrocution}

In this subsection, we indicate, following [22], a modification of Farb's [10] notion of strong relative hyperbolicity and his construction of an electric metric, described earlier. The modification we shall discuss is called partial electrocution and will be used in proving the converse to the Strong Combination Theorem (Theorem 4.5). Most of this discussion is taken from [22].

We start with a few motivating examples:

Partial electrocution of a horosphere $H=\mathbb{R}^{n-1} \times \mathbb{R}$ will be defined as putting the zero metric in the $\mathbb{R}^{n-1}$ direction, and retaining the usual Euclidean metric in the other $\mathbb{R}$ direction.

In the partially electrocuted case, instead of coning all of a horosphere down to a point we cone only horocyclic leaves of a foliation of the horosphere. Effectively, therefore, we have a cone-line rather a cone-point.

Let $Y$ be a convex simply connected hyperbolic $n$-manifold. Let $\mathcal{B}$ denote a collection of horoballs. Let $X$ denote $Y$ minus the interior of the horoballs in $\mathcal{B}$. Let $\mathcal{H}$ denote the collection of boundary horospheres. Then each $H \in \mathcal{H}$ with the induced metric is isometric to a Euclidean product $E^{n-2} \times L$ for an interval $L \subset \mathbb{R}$. Partially electrocute each $H$ by giving it the product of the zero metric with the Euclidean metric, ie on $E^{n-2}$ give the zero metric and on $L$ give the Euclidean metric. The resulting space is exactly what one would get by gluing to each $H$ the mapping cylinder of the projection of $H$ onto the $L$-factor. 
This motivates the following scenario:

Definition 2.9 Let $(X, \mathcal{H}, \mathcal{G}, \mathcal{L})$ be an ordered quadruple such that the following holds:

(1) $X$ is (strongly) hyperbolic relative to a collection of subsets $H_{\alpha}$, thought of as horospheres (and not horoballs).

(2) For each $H_{\alpha}$ there is a uniform large-scale retraction $g_{\alpha}: H_{\alpha} \rightarrow L_{\alpha}$ to some (uniformly) $\delta$-hyperbolic metric space $L_{\alpha}$, ie there exist $\delta, K, \epsilon>0$ such that for all $H_{\alpha}$ there exists a $\delta$-hyperbolic $L_{\alpha}$ and a map $g_{\alpha}: H_{\alpha} \rightarrow L_{\alpha}$ with $d_{L_{\alpha}}\left(g_{\alpha}(x), g_{\alpha}(y)\right) \leq K d_{H_{\alpha}}(x, y)+\epsilon$ for all $x, y \in H_{\alpha}$. Further, we denote the collection of such $g_{\alpha}$ 's as $\mathcal{G}$.

The partially electrocuted space or partially coned-off space corresponding to the quadruple $(X, \mathcal{H}, \mathcal{G}, \mathcal{L})$ is obtained from $X$ by gluing in the (metric) mapping cylinders for the maps $g_{\alpha}: H_{\alpha} \rightarrow L_{\alpha}$.

In Farb's construction $L_{\alpha}$ is just a single point. However, the notions and arguments of Farb [10] or Klarreich [17] go through even in this setting. The metric, and geodesics and quasigeodesics in the partially electrocuted space will be referred to as the partially electrocuted metric $d_{\mathrm{pel}}$, and partially electrocuted geodesics and quasigeodesics respectively. In this situation, we conclude as in Lemma 2.4:

Lemma 2.10 $\left(X, d_{\text {pel }}\right)$ is a hyperbolic metric space and the sets $L_{\alpha}$ are uniformly quasiconvex.

Note 1 When $K_{\alpha}$ is a point, the last statement is a triviality.

Note $2\left(X, d_{\text {pel }}\right)$ is strongly hyperbolic relative to the sets $\left\{L_{\alpha}\right\}$. In fact the space obtained by electrocuting the sets $L_{\alpha}$ in $\left(X, d_{\text {pel }}\right)$ is just the space $\left(X, d_{e}\right)$ obtained by (completely) electrocuting the sets $\left\{H_{\alpha}\right\}$ in $X$.

Note 3 The proof of Lemma 2.10 and other such results below follow Farb's [10] constructions. For instance, consider a hyperbolic geodesic $\eta$ in a convex complete simply connected $n$-manifold $X$ with pinched negative curvature. Let $H_{i}, i=1 \cdots k$ be the partially electrocuted horoballs it meets. Let $N(\eta)$ denote the union of $\eta$ and $H_{i}$ 's. Let $Y$ denote $X$ minus the interiors of the $H_{i}$ 's. The first step is to show that $N(\eta) \cap Y$ is quasiconvex in $\left(Y, d_{\text {pel }}\right)$. To do this one takes a hyperbolic $R-$ neighborhood of $N(\eta)$ and projects $\left(Y, d_{\text {pel }}\right)$ onto it, using the hyperbolic projection. It was shown by Farb in [10] that the projections of all horoballs are uniformly bounded 
in hyperbolic diameter. (This is essentially mutual coboundedness). Hence, given $K$, choosing $R$ large enough, any path that goes out of an $R$-neighborhood of $N(\eta)$ cannot be a $K$-partially electrocuted quasigeodesic. This is the one crucial step that allows the results of [10], in particular, Lemma 2.10 to go through in the context of partially electrocuted spaces.

As in Lemma 2.7, partially electrocuted quasigeodesics and geodesics without backtracking have the same intersection patterns with horospheres and boundaries of lifts of tubes as electric geodesics without backtracking. Further, since electric geodesics and hyperbolic quasigeodesics have similar intersection patterns with horoballs and lifts of tubes it follows that partially electrocuted quasigeodesics and hyperbolic quasigeodesics have similar intersection patterns with horospheres and boundaries of lifts of tubes. We state this formally below:

Lemma 2.11 Given $K, \epsilon \geq 0$, there exists $C>0$ such that the following holds:

Let $\gamma_{\mathrm{pel}}$ and $\gamma$ denote respectively a $(K, \epsilon)$ partially electrocuted quasigeodesic in $\left(X, d_{\text {pel }}\right)$ and a hyperbolic $(K, \epsilon)$-quasigeodesic in $(Y, d)$ joining $a, b$. Then $\gamma \cap X$ lies in a (hyperbolic) $C$-neighborhood of (any representative of) $\gamma_{\text {pel }}$. Further, outside of a $C$-neighborhood of the horoballs that $\gamma$ meets, $\gamma$ and $\gamma_{\text {pel }}$ track each other.

\section{Trees of hyperbolic metric spaces}

\subsection{Trees of spaces: hyperbolic and relatively hyperbolic}

We start with a notion closely related to one introduced in [4].

Definition 3.1 A tree (T) of hyperbolic (resp. strongly relatively hyperbolic) metric spaces satisfying the q(uasi) i(sometrically) embedded condition is a metric space $(X, d)$ admitting a map $P: X \rightarrow T$ onto a simplicial tree $T$, such that there exist $\delta, \epsilon$ and $K>0$ satisfying the following:

(1) For all vertices $v \in T, X_{v}=P^{-1}(v) \subset X$ with the induced path metric $d_{v}$ is a $\delta$-hyperbolic metric space (resp. a geodesic metric space $X_{v}$ strongly hyperbolic relative to a collection $\mathcal{H}_{v \alpha}$ ). Further, the inclusions $i_{v}: X_{v} \rightarrow X$ are uniformly proper, ie for all $M>0, v \in T$ and $x, y \in X_{v}$, there exists $N>0$ such that $d\left(i_{v}(x), i_{v}(y)\right) \leq M$ implies $d_{v}(x, y) \leq N$.

(2) Let $e$ be an edge of $T$ with initial and final vertices $v_{1}$ and $v_{2}$ respectively. Let $X_{e}$ be the preimage under $P$ of the midpoint of $e$. Then $X_{e}$ with the induced path metric is $\delta$-hyperbolic (resp. a geodesic metric space $X_{e}$ strongly hyperbolic relative to a collection $\left.\mathcal{H}_{e \alpha}\right)$. 
(3) There exist maps $f_{e}: X_{e} \times[0,1] \rightarrow X$, such that $\left.f_{e}\right|_{X_{e} \times(0,1)}$ is an isometry onto the preimage of the interior of $e$ equipped with the path metric.

(4) $\left.f_{e}\right|_{X_{e} \times\{0\}}$ and $\left.f_{e}\right|_{X_{e} \times\{1\}}$ are $(K, \epsilon)$-quasi-isometric embeddings into $X_{v_{1}}$ and $X_{v_{2}}$ respectively. $\left.f_{e}\right|_{X_{e} \times\{0\}}$ and $\left.f_{e}\right|_{X_{e} \times\{1\}}$ will occasionally be referred to as $f_{v_{1}}$ and $f_{v_{2}}$ respectively.

(5) For a tree of strongly relatively hyperbolic spaces, we demand in addition, that the maps $f_{v_{i}}$ above $(i=1,2)$ are strictly type-preserving, ie $f_{v_{i}}^{-1}\left(H_{v_{i} \alpha}\right), i=1,2$ (for any $H_{v_{i} \alpha} \in \mathcal{H}_{v_{i} \alpha}$ ) is either empty or some $H_{e \alpha} \in \mathcal{H}_{e \alpha}$.

(6) For a tree of strongly relatively hyperbolic spaces, we demand that the coned-off spaces are uniformly $\delta$-hyperbolic.

Denote by $d_{v}$ and $d_{e}$ path metrics on $X_{v}$ and $X_{e}$ respectively. $i_{v}, i_{e}$ will denote inclusion of $X_{v}, X_{e}$ respectively into $X$.

For a tree of relatively hyperbolic spaces, the sets $H_{v \alpha}$ and $H_{e \alpha}$ will be referred to as horosphere-like vertex sets and edge sets respectively.

When $(X, d)$ is a tree $(\mathrm{T})$ of strongly relatively hyperbolic metric spaces, the strictly type-preserving condition (Condition 5 above) ensures that we obtain an induced tree (T) (the same tree $\mathrm{T}$ ) of coned-off, or electric spaces. We demand further:

Qi-preserving electrocution condition The induced maps of the electric edge spaces into the electric vertex spaces $\widehat{f_{v_{i}}}: \widehat{X_{e}} \rightarrow \widehat{X_{v_{i}}}(i=1,2)$ are uniform quasi-isometries.

The resulting tree of coned-off spaces will be called the induced tree of coned-off spaces. The resulting space will be denoted as $\widehat{X}$.

Definition 3.2 A finite graph of (strongly) relatively hyperbolic groups is said to satisfy Condition $C$, if the associated tree of relatively hyperbolic Cayley graphs satisfies Condition $C$. Here $C$ will be one of the following:

(1) the qi-embedded condition

(2) the strictly type-preserving condition

(3) the qi-preserving electrocution condition

(4) the induced tree of coned-off spaces satisfies the hallways flare condition (see Definition 3.5)

(5) the cone-bounded hallways strictly flare condition (see Definition 3.6). 
Remark Strictly speaking, this induced tree exists for any collection of vertex and edge spaces satisfying the strictly type-preserving condition. Hyperbolicity is not essential for the existence of the induced tree of spaces.

The cone locus of $\widehat{X}$, the induced tree (T) of coned-off spaces, is the graph (in fact a forest) whose vertex set $\mathcal{V}$ consists of the cone-points in the vertex set of $\widehat{X}$ and whose edge-set $\mathcal{E}$ consists of the cone-points in the edge set of $\widehat{X}$. The incidence relations are dictated by the incidence relations in $T$.

Note that connected components of the cone-locus can be naturally identified with subtrees of $T$. Each such connected component of the cone-locus will be called a maximal cone-subtree. The collection of maximal cone-subtrees will be denoted by $\mathcal{T}$ and elements of $\mathcal{T}$ will be denoted as $T_{\alpha}$. Further, each maximal cone-subtree $T_{\alpha}$ naturally gives rise to a tree $T_{\alpha}$ of horosphere-like subsets depending on which conepoints arise as vertices and edges of $T_{\alpha}$. The metric space that $T_{\alpha}$ gives rise to will be denoted as $C_{\alpha}$ and will be referred to as a maximal cone-subtree of horosphere-like spaces. The collection of $C_{\alpha}$ 's will be denoted as $\mathcal{C}$.

Note Each $T_{\alpha}$ thus appears in two guises:

(1) as a subset of $\widehat{X}$

(2) as the underlying tree of $C_{\alpha}$

We shall have need for both these interpretations.

\subsection{The Bestvina-Feighn flare condition}

Next, we would like to recall the essential condition (due to Bestvina and Feighn [4]) ensuring hyperbolicity of a tree of spaces. We retain the terminology.

Definition 3.3 A disk $f:[-m, m] \times I \rightarrow X$ is a hallway of length $2 m$ if it satisfies:

(1) $f^{-1}\left(\cup X_{v}: v \in T\right)=\{-m, \cdots, m\} \times I$.

(2) $f$ maps $i \times I$ to a geodesic in $X_{v}$ for some vertex space.

(3) $f$ is transverse, relative to condition (1) to $\cup_{e} X_{e}$.

Definition 3.4 A hallway is $\rho$-thin if $d(f(i, t), f(i+1, t)) \leq \rho$ for all $i, t$.

A hallway is $\lambda$-hyperbolic if

$$
\lambda l(f(\{0\} \times I)) \leq \max \{l(f(\{-m\} \times I)), l(f(\{m\} \times I)) .
$$


A hallway is essential if the edge path in $T$ resulting from projecting $X$ onto $T$ does not backtrack (and is therefore a geodesic segment in the tree $T$ ).

An essential hallway of length $2 m$ is cone-bounded if $f(i \times \partial I)$ lies in the cone-locus for $i=\{-m, \cdots, m\}$.

Definition 3.5 (Hallways flare condition) The tree of spaces, $X$, is said to satisfy the hallways flare condition if there are numbers $\lambda>1$ and $m \geq 1$ such that for all $\rho$ there is a constant $H(\rho)$ such that any $\rho$-thin essential hallway of length $2 m$ and girth at least $H$ is $\lambda$-hyperbolic.

Definition 3.6 (Cone-bounded hallways strictly flare condition) The tree of spaces, $X$, is said to satisfy the hallways flare condition if there are numbers $\lambda>1$ and $m \geq 1$ such that any cone-bounded hallway of length $2 m$ is $\lambda$-hyperbolic.

The main theorem of Bestvina and Feighn follows (though this is stated in [4] for groups, the proof nowhere requires uniform properness of the space). Bowditch [7] notes the equivalence of the hallways flare condition with the hyperbolicity of the tree of spaces.

Theorem 3.7 [4, pp 85-86; 7] Let $X$ be a tree of hyperbolic metric spaces satisfying the qi-embedded condition and the hallways flare condition. Then $X$ is hyperbolic.

Conversely, if $X$ is hyperbolic, then hallways flare.

Apart, from Theorem 3.7 above, we shall need one more simple observation.

Lemma 3.8 Suppose that $\widehat{X}$ is hyperbolic. Then maximal cone-subtrees $T_{\alpha}$ are uniformly quasiconvex in $\widehat{X}$.

Proof Let $P: X \rightarrow T$ be the natural projection map of the tree of spaces to the underlying subtree. Then $P$ induces $P^{\prime}: \widehat{X} \rightarrow T$ as $\widehat{X}$ may be regarded as (the same) tree (T) of coned-off spaces. $P^{\prime}$ is distance nonincreasing. Further, restricted to each $T_{\alpha}, P^{\prime}$ is an isometry.

Also note that any path from $x \in H_{v 1}$ to $y \in H_{v 2}$ in $\widehat{X}$ has length not less than $d_{T}\left(P^{\prime}(x), P^{\prime}(y)\right)$, where $d_{T}$ is the natural metric on $T$ and $v_{1}, v_{2} \in T_{\alpha}$.

Now suppose that $x, y \in T_{\alpha} \subset \widehat{X}$. Let $\gamma \subset T_{\alpha} \widehat{X}$ be the geodesic in $T_{\alpha}$ joining $x, y$. It therefore follows that for any $x, y \in T_{\alpha} \subset \widehat{X}$ and any path $A$ joining $x, y \in \widehat{X}$,

$$
l(A) \leq d_{T}\left(P^{\prime}(x), P^{\prime}(y)\right)=l(\gamma) .
$$

Hence $\gamma$ is quasi-isometrically (in fact isometrically) embedded in $\widehat{X}$ and hence a geodesic in $\widehat{X}$. The Lemma follows. 


\section{The Combination Theorem}

\subsection{Weak Combination Theorem}

We start with the following:

Theorem 4.1 (Weak Combination Theorem) Let $X$ be a tree ( $T$ ) of strongly relatively hyperbolic spaces satisfying:

(1) the qi-embedded condition

(2) the strictly type-preserving condition

(3) the qi-preserving electrocution condition

(4) the induced tree of coned-off spaces satisfies the hallways flare condition.

Then $X$ is weakly hyperbolic relative to the family $\mathcal{C}$ of maximal cone-subtrees of horosphere-like spaces.

Proof As usual let $\widehat{X}$ denote the induced tree $(T)$ of coned-off spaces, $\mathcal{T}$ denote the family of maximal cone-subtrees $T_{\alpha} \subset \widehat{X}$. Let $\widehat{X}^{c}$ denote $\widehat{X}$ with the family of sets $\mathcal{T}$ coned off (ie vertices $v_{\alpha}$ are introduced, one each for each $T_{\alpha}$, and joined to points of the corresponding $T_{\alpha}$ by edges of length $1 / 2$ ).

Since vertex and edge-spaces are strongly relatively hyperbolic, then by item (6) in the definition of a tree of strongly relatively hyperbolic spaces, $\widehat{X}$ is a tree of (uniformly) hyperbolic metric spaces.

By the qi-preserving electrocution condition, the induced tree of coned-off spaces satisfies the qi-embedded condition.

By the hallways flare condition and Theorem 3.7, $\widehat{X}$ is a hyperbolic metric space.

By Lemma 3.8, the sets $T_{\alpha} \in \mathcal{T}$ are uniformly quasiconvex and uniformly separated.

Hence by Lemma $2.4, \widehat{X}$ is weakly hyperbolic relative to the sets $T_{\alpha} \in \mathcal{T}$, ie $\widehat{X}^{c}$ is a hyperbolic metric space.

Let $\widehat{X_{1}}$ denote the space obtained from $X$ by coning off maximal cone-subtrees of horosphere-like sets. Then $\widehat{X_{1}}$ is quasi-isometric to $\widehat{X}^{c}$. To see this, one notes that $\widehat{X}^{c}$ is obtained from $X$ by first coning-off (or partially electrocuting) $C_{\alpha}$ 's, the maximal cone-subtrees of horosphere-like sets, to maximal cone-subtrees $T_{\alpha}$. This gives rise to $\widehat{X}$. Further coning off the $T_{\alpha}$ 's gives $\widehat{X}^{c}$. On the other hand, $\widehat{X}_{1}$ is obtained from $X$ by coning off (or completely electrocuting) the $C_{\alpha}$ 's to points in one step. The two constructions clearly give quasi-isometric spaces.

Hence $\widehat{X_{1}}$ is hyperbolic, ie $X$ is weakly hyperbolic relative to the collection of sets $C_{\alpha} \in \mathcal{C}$. 


\subsection{Strong Combination Theorem}

Under the additional cone-bounded hallways strictly flare condition, we would now like to prove a stronger version of the combination Theorem 4.1, ie $X$ is strongly hyperbolic relative to the collection of $C_{\alpha} \in \mathcal{C}$ :

By Lemma 2.7 and Theorem 4.1, it suffices to show that the sets $T_{\alpha} \subset \widehat{X}$ are mutually cobounded. Most of the rest of this subsection is devoted to proving mutual coboundedness.

The next Lemma follows easily from stability of quasigeodesics [12] [14]. (See, for instance Lemma 4.1.1 of [18].)

Lemma 4.2 Give $\delta, C$, there exist $D, K, \epsilon$ such that the following holds:

Let $(X, d)$ be a $\delta$-hyperbolic metric space and $Y$ a $C$-quasiconvex subset. Let $\pi$ be a nearest-point retraction of $X$ onto $Y$. Let $x, y \in X$ such that $d(\pi(x), \pi(y)) \geq D$. Then $[x, \pi(x)] \cup[\pi(x), \pi(y)] \cup[\pi(y), y]$ is a $(K, \epsilon)$-quasigeodesic.

We use Lemma 4.2 below:

Corollary 4.3 Given $\delta, C$, there exist $D, M$ such that the following holds:

Suppose that $Y, Z$ are $C$-quasiconvex subsets of a $\delta$-hyperbolic metric space $(X, d)$. Let $\pi$ denote nearest point projection onto $Y$. If $\pi(Z)$ has diameter greater than $D$, then $\pi(Z)$ lies in an $M$-neighborhood of $Z$.

Proof Let $x, y \in Z$. By Lemma 4.2, there exist $D_{0}, K, \epsilon$ (depending on $\delta, C$ ) such that if $d(\pi(x), \pi(y)) \geq D_{0}$, then $[x, \pi(x)] \cup[\pi(x), \pi(y)] \cup[\pi(y), y]=\gamma$ is a $(K, \epsilon)$-quasigeodesic. Since $Z$ is $C$-quasiconvex, $\gamma$ lies in an $M_{0}=M_{0}(K, \epsilon, C, \delta)$ neighborhood of $Z$.

Now, choose $a, b$ in $Y, Z$ respectively, such that $d(a, b)=d(Y, Z)$. From the previous paragraph, we deduce that if $z \in Z$ such that $d(\pi(z), a) \geq D_{0}$, then $\pi(z)$ lies in an $M_{0}$-neighborhood of $Z$. Taking $D=2 D_{0}$ and $M=M_{0}+D_{0}$, we are through.

The following Proposition deduces mutual coboundedness of maximal cone-subtrees $T_{\alpha}$ in $\widehat{X}$ (obtained by partially electrocuting maximal cone-subtrees of horosphere-like sets) from hyperbolicity of $\widehat{X}$ (established for instance in Theorem 4.1) and conebounded hallways strictly flare condition. This will be used in proving the Strong Combination Theorem (Theorem 4.5). 
Proposition 4.4 Suppose that the tree of coned-off spaces $\widehat{X}$ is hyperbolic and that the cone-bounded hallways strictly flare condition is satisfied. Then there exists $D \geq 0$ such that the family of maximal cone-subtrees $T_{\alpha}$ in $\widehat{X}$ is $D$-cobounded.

Proof Suppose not. Then, by Corollary 4.3, there exists $M \geq 0$ such that for any $D \geq 0$, there exist maximal cone-subtrees $T_{1}, T_{2}$ and a connected subtree $T_{3} \subset T_{1}$ such that $T_{3}$ has diameter greater than $D$ and lies in an $M$-neighborhood of $T_{2}$. Hence, there exist geodesic edge-paths $\gamma_{1} \subset T_{1}$ and $\gamma_{2} \subset T_{2}$ such that each $\gamma_{i}$ has length greater than $(D-2 M)$ and such that they lie in an $M$-neighborhood of each other in $\widehat{X}$.

Next, $P\left(\gamma_{i}\right) \subset T$ (where $T$ is the tree underlying $\widehat{X}$, the tree (T) of coned-off spaces and $P: \widehat{X} \rightarrow T$ is the natural projection). Also, $P\left(\gamma_{i}\right)(i=1,2)$ is abstractly isomorphic to $\gamma_{i}$ as an edge-path, since $P: \widehat{X} \rightarrow T$ is an isometry restricted to each $T_{i}$. Then $P\left(\gamma_{i}\right)$ may be regarded as geodesic paths in $T$ having lengths greater than $(D-2 M)$ and lying in an $M$-neighborhood of each other (since $P$ does not increase distances). This means that $P\left(\gamma_{i}\right)$ (for $i=1,2$ ) must overlap over an interval of length at least $(D-4 M)$.

Let $\alpha_{i} \subset \gamma_{i}$ be paths having length at least $(D-4 M)$ with $P\left(\alpha_{1}\right)=P\left(\alpha_{2}\right)$. Then there exists $M_{1}=M_{1}(M)$ and a cone-bounded hallway $\Delta:[-m, m] \times I \rightarrow \widehat{X}$ with $2 m \geq(D-4 M)$ such that:

(1) $\Delta\left([-m, m] \times\{0\}=\alpha_{1}\right.$

(2) $\Delta\left([-m, m] \times\{1\}=\alpha_{2}\right.$

(3) each $\Delta(j \times I)$ has length less than $M_{1}$.

Since $D$, and hence $m$ can be arbitrarily large, while $M$ (and hence $M_{1}$ ) are fixed,it follows that for any given $\lambda>1$, there exists a hallway $\Delta$, which is not $\lambda$-hyperbolic. This violates the cone-bounded hallways strictly flare condition. Hence, by contradiction, there exists $D \geq 0$ such that the family of maximal cone-subtrees $T_{\alpha}$ in $\widehat{X}$ is $D$-cobounded.

We are now in a position to prove:

Theorem 4.5 (Strong Combination Theorem) Let $X$ be a tree $(T)$ of strongly relatively hyperbolic spaces satisfying:

(1) the qi-embedded condition

(2) the strictly type-preserving condition 
(3) the qi-preserving electrocution condition

(4) the induced tree of coned-off spaces satisfies the hallways flare condition

(5) the cone-bounded hallways strictly flare condition.

Then $X$ is strongly hyperbolic relative to the family $\mathcal{C}$ of maximal cone-subtrees of horosphere-like spaces.

Proof By Theorem 4.1, we know that $X$ is weakly hyperbolic relative to the family $\mathcal{C}$ of maximal cone-subtrees of horosphere-like spaces.

This is equivalent to saying that $\widehat{X}$ is weakly hyperbolic relative to the family $\mathcal{T}$ of maximal cone-subtrees $T_{\alpha} \subset \widehat{X}$.

By the cone-bounded hallways strictly flare condition and Proposition 4.4, we see that the family $\mathcal{T}$ is mutually cobounded.

Hence by Lemma 2.7, we conclude that $\widehat{X}$ is strongly hyperbolic relative to the family $\mathcal{T}$ of maximal cone-subtrees $T_{\alpha} \subset \widehat{X}$. Equivalently, $X$ is strongly hyperbolic relative to the family $\mathcal{C}$ of maximal cone-subtrees of horosphere-like spaces.

Recall that a finite graph of (strongly) relatively hyperbolic groups is said to satisfy a Condition $C$, if the associated tree of relatively hyperbolic Cayley graphs also satisfies Condition $C$. The resulting group will be denoted as $G$. A quotient of maximal cone-subtrees of horosphere-like spaces in this case, is called a maximal cone-subgraph of horosphere-like subgroups. Note that such a subgraph gives rise to a subgroup of $G$. We shall refer to such subgroups as maximal parabolic subgroups. Recall that we are using the convention that all parabolic subgroups are of infinite index. As an immediate consequence of Theorem 4.5, we have the following:

Theorem 4.6 (Strong Combination Theorem for graphs of groups) Let $G$ be a finite graph $(\Gamma)$ of strongly relatively hyperbolic groups satisfying:

(1) the qi-embedded condition

(2) the strictly type-preserving condition

(3) the qi-preserving electrocution condition

(4) the induced tree of coned-off spaces satisfies the hallways flare condition

(5) the cone-bounded hallways strictly flare condition.

Then $G$ is strongly hyperbolic relative to the family $\mathcal{C}$ of maximal parabolic subgroups. 


\subsection{Converse to the Strong Combination Theorem}

Recall that the partially electrocuted space or partially coned-off space corresponding to a quadruple $(X, \mathcal{H}, \mathcal{G}, \mathcal{L})$ is obtained from $X$ by gluing in the (metric) mapping cylinders for the maps $g_{\alpha}: H_{\alpha} \rightarrow L_{\alpha}$. Note that from Theorem 4.5, it follows that $\widehat{X}$ is obtained from $X$ by partially electrocuting each $C_{\alpha}$. Here:

(1) $H_{\alpha}=C_{\alpha}$ and $\mathcal{H}=\mathcal{C}$

(2) $L_{\alpha}=T_{\alpha}$ and $\mathcal{L}=\mathcal{T}$

(3) $g_{\alpha}: C_{\alpha} \rightarrow T_{\alpha}$ collapses $C_{\alpha}$, the tree of horosphere-like spaces to the underlying tree $T_{\alpha}$.

Theorem 4.7 (Converse to Strong Combination Theorem) Let $X$ be a tree ( $T$ ) of strongly relatively hyperbolic spaces satisfying:

(1) the qi-embedded condition

(2) the strictly type-preserving condition

(3) the qi-preserving electrocution condition

(4) $X$ is strongly hyperbolic relative to the family $\mathcal{C}$ of maximal cone-subtrees of horosphere-like spaces.

Then the induced tree of coned-off spaces satisfies the hallways flare condition and the cone-bounded hallways strictly flare condition.

Proof As usual let $C_{\alpha}$ denote maximal cone subtrees $\left(T_{\alpha}\right)$ of horosphere-like sets. By Lemma 2.10, the induced tree of coned-off spaces $\widehat{X}$, obtained by partially electrocuting each $C_{\alpha}$ to $T_{\alpha}$. Then by the converse part of Theorem 3.7, hallways, including conebounded hallways flare.

It remains to show that cone-bounded hallways strictly flare. Suppose not. Then there exists $D_{0}$ such that for all $N \in \mathbb{N}$, there exist cone-bounded hallways of length greater than $N$, bounded by "vertical" (parametrized) geodesics $\lambda_{1}, \lambda_{2}$ in distinct cone-subtrees $T_{1}, T_{2}$ respectively such that $d\left(\lambda_{1}(i), \lambda_{2}(i)\right) \leq D_{0}$ for all $i=0, \cdots N$. Let $\mu_{0}, \mu_{N}$ denote "horizontal" paths in the hallway joining $\lambda_{1}(i), \lambda_{2}(i)$ for $i=0, N$. Hence, there exist points $a_{j}, b_{j}(j=0, N)$ lying on the corresponding cones $C_{1} \cap \mu_{j}, C_{2} \cap \mu_{j}$, respectively such that $d\left(a_{j}, b_{j}\right) \leq D_{0}$. Then we have two paths: 
- $\sigma_{1}$ starts at $a_{0}$, moves to $\lambda_{1}(0)$ (by a cone-edge of length $1 / 2$ ), proceeds to $\lambda_{1}(N)$ and exits to $a_{N}$ (again by a cone-edge of length $1 / 2$ ).

- $\sigma_{2}$ starts at $a_{0}$, moves to $b_{0}$ by a path of length $\leq D_{0}$, then to $\lambda_{2}(0)$ (by a cone-edge of length $1 / 2$ ), proceeds to $\lambda_{2}(N)$, exits to $b_{N}$ (again by a cone-edge of length $1 / 2$ ) and then goes to $a_{N}$ by a path of length $\leq D_{0}$.

The path $\sigma_{1}$ has length 1 in the electric metric on $\widehat{X}^{c}$ and $\sigma_{2}$ has length $\leq 2 D_{0}+1$. Since $D_{0}$ is fixed, both $\sigma_{1}$ and $\sigma_{2}$ are uniform quasigeodesics beginning and ending at the same point, but have manifestly different intersection patterns with $C_{1}, C_{2}$. This contradicts Lemma 2.7 and hence $X$ cannot be strongly hyperbolic relative to the collection $C_{\alpha}$. This final contradiction proves the Theorem.

\subsection{Examples}

The first class of examples are hyperbolic 3-manifolds fibering over the circle with fiber a punctured hyperbolic surface $\Sigma$. All the conditions of Theorem 4.6 are satisfied in this case of a 3-manifold fibering over the circle with fiber a punctured surface. The one condition that needs checking is the hallways flare condition for the induced tree (in fact line) of coned-off spaces. This fact is due to Bowditch [7, Section 6]. We give here a somewhat different argument based on work of Mosher [26].

In [26], Mosher constructs examples of exact sequences of hyperbolic groups of the form

$$
1 \rightarrow H \rightarrow G \rightarrow F \rightarrow 1
$$

where $H$ is a closed surface group, $G$ is hyperbolic and $F$ is free. (This construction is modified by Bestvina, Feighn and Handel [5] to the case where $H$ is a free group.)

We shall modify Mosher's argument slightly to make it work for punctured surfaces.

Let $\Phi$ be a pseudo-Anosov diffeomorphism of a punctured hyperbolic surface $\Sigma$. Taking a suitable power of $\Phi$ if necessary, we may assume that $\Phi$ fixes all the punctures. The stable and unstable foliations of $\Phi$ give rise to a piecewise Euclidean metric on $\Sigma$. This metric is incomplete at the punctures. Complete it to get a surface with boundary $\Sigma_{B}$, which may be thought of as the blow-up of $\Sigma$ at the punctures. Equip $\Sigma_{B}$ with a pseudometric which is zero on all the boundary components and equal to the piecewise Euclidean metric elsewhere. This metric is discontinuous at the boundary, but this is not important. This is essentially the electric metric on $\Sigma_{B}$.

Then any electric geodesic $\lambda$ in $\Sigma_{B}$ is the union of two types of segments:

(1) geodesics in the piecewise Euclidean metric meeting the boundary at right angles. Let $\lambda_{\text {eu }}$ denote this union.

(2) segments lying along the boundary. 
The total length of such an electric geodesic is the sum of the lengths of the Euclidean pieces. The projection of the union of the Euclidean pieces $\lambda_{\text {eu }}$ onto the stable and unstable foliations will be denoted by $\lambda_{\text {eus }}$ and $\lambda_{\text {euu }}$ respectively. Abusing notation slightly, we assume that $\lambda_{\text {eu }}, \lambda_{\text {eus }}, \lambda_{\text {euu }}$ denote the respective lengths also. Then $\max \left(\lambda_{\text {eus }}, \lambda_{\text {euu }}\right) \geq 1 / 2 \lambda_{\text {eu }}$. Let $\Phi\left(\lambda_{\text {eu }}\right)$ denote the image of $\lambda_{\text {eu }}$ under $\Phi$. If we assume that the stable and unstable foliations meet the boundary components of $\Sigma_{B}$ at right angles, then it can be easily shown that for any given $k>1$, there is an $n$ (depending on the stretch factor of $\Phi$ ) such that

$$
\max \left(\Phi\left(\lambda_{\text {eus }}\right), \Phi^{-1}\left(\lambda_{\text {euu }}\right)\right) \geq k \lambda_{\text {eu }}
$$

and hence we have the inequality

$$
\max \left(\Phi\left(\lambda_{\mathrm{eu}}\right), \Phi^{-1}\left(\lambda_{\mathrm{eu}}\right)\right) \geq k \lambda_{\mathrm{eu}}
$$

This proves the one condition that needed checking, viz. the hallways flare condition for the induced tree (in fact line) of coned-off spaces.

More generally, we may take any $m$ (equal to two below for concreteness) pseudoAnosov diffeomorphisms $\Phi, \Psi$ with different stable and unstable foliations. Then generalizing the above construction, we can prove the following generalization of an essential Lemma of Mosher: 3 out of 4 stretch.

Lemma 4.8 For any $k>1$, there exists $n>0$ such that for any electric geodesic $\lambda$ in $\Sigma_{B}$, at least three of the four elements $\Phi^{n}, \Phi^{-n}, \Psi^{n}, \Psi^{-n}$ stretch $\lambda$ by a factor of $\lambda$.

Thus we get an exact sequence of groups of the form

$$
1 \rightarrow H \rightarrow G \rightarrow F \rightarrow 1
$$

where $H$ is a punctured surface group, and $F$ is free.

The Cayley graph of $G$ may thus be regarded as a tree $T$ of hyperbolic spaces, where $T$ arises as the Cayley graph of $F$. Also, the maximal parabolic subgroups here correspond exactly to the peripheral subgroups, ie the cusp groups. Maximal cone-subtrees are each isometric to $T$. Then a tree $T$ of maximal parabolics would correspond to $\mathbb{Z} \times F$. Lemma 4.8 shows that the induced tree of coned-off spaces is hyperbolic.

Thus, we obtain from Theorem 4.6: 
Theorem 4.9 Let $\Phi_{1} \cdots \Phi_{m}$ be $m$ (orientation-preserving) pseudo-Anosov diffeomorphisms of $\Sigma$ with different sets of stable and unstable foliations. Let $H=\pi_{1}(\Sigma)$. Then there is an $n \geq 1$ such that the diffeomorphisms $\Phi_{1}^{n}, \cdots \Phi_{m}^{n}$ generate a free group $F$ and the group $G$ given by the exact sequence

$$
1 \rightarrow H \rightarrow G \rightarrow F \rightarrow 1
$$

is (strongly) hyperbolic relative to the maximal parabolic subgroups of the form $\mathbb{Z} \times F$.

For $n=1$, we get back Bowditch's theorem [7].

\subsection{Applications, consequences and problems}

Theorem 4.5 and Theorem 4.6 open up the possibility of generalizing several theorems about hyperbolic groups to (strongly) relatively hyperbolic groups.

Cannon-Thurston maps In [19], the first author proved the existence of CannonThurston maps for trees of hyperbolic metric spaces. In [21], he generalized this theorem to the relatively hyperbolic case under the additional assumption that the tree of spaces gives rise to a hyperbolic 3-manifold of bounded geometry whose core is incompressible away from cusps. In [24], $\mathrm{Mj}-\mathrm{Pal}$ prove the existence of CannonThurston maps for the situation discussed in this paper, viz. trees of (strongly) relatively hyperbolic trees of metric spaces that are (strongly) relatively hyperbolic.

Strongly relatively hyperbolic extensions of groups A Theorem of Mosher [25] says that if an exact sequence of groups of the form

$$
1 \rightarrow H \rightarrow G \rightarrow Q \rightarrow 1
$$

exists, where $H$ is hyperbolic, then there exists a quasi-isometric section of $\Gamma_{Q}$ into $\Gamma_{G}$ exists. In particular, if $G$ is hyperbolic, so is $Q$. The essential technique is to use the action of $Q$ on the boundary $\partial H$ of $H$.A fact (due to Gromov [14]) that is used is that the space of triples of points on the boundary of a hyperbolic group $H$ is quasi-isometric to $\Gamma_{H}$. An analogous result is shown by Pal in [27].

Heights of groups In [13], Gitik, Mitra, Rips and Sageev show that quasiconvex subgroups of hyperbolic groups have finite height and finite width. A partial converse was obtained by the first author in [20] for groups splitting over subgroups. This converse was used by Swarup in [29] to prove a weak hyperbolization theorem. All three theorems should have analogues in the (strongly) relatively hyperbolic world. Hruska and Wise [15] have already shown the finiteness of height and width of quasiconvex subgroups of relatively hyperbolic groups. 


\section{References}

[1] E Alibegović, A combination theorem for relatively hyperbolic groups, Bull. London Math. Soc. 37 (2005) 459-466 MR2131400

[2] J Behrstock, C Drutu, L Mosher, Thick metric spaces, relative hyperbolicity, and quasi-isometric rigidity arXiv:math/0512592

[3] M Bestvina, Geometric group theory problem list, available at http://math.utah.edu/ bestvina

[4] M Bestvina, M Feighn, A combination theorem for negatively curved groups, J. Differential Geom. 35 (1992) 85-101 MR1152226

[5] M Bestvina, M Feighn, M Handel, Laminations, trees, and irreducible automorphisms of free groups, Geom. Funct. Anal. 7 (1997) 215-244 MR1445386

[6] B H Bowditch, Relatively hyperbolic groups, preprint, Southampton (1997)

[7] B H Bowditch, The Cannon-Thurston map for punctured-surface groups, Math. Z. 255 (2007) 35-76 MR2262721

[8] I Bumagin, On definitions of relatively hyperbolic groups, from: "Geometric methods in group theory", Contemp. Math. 372, Amer. Math. Soc. (2005) 189-196 MR2139687

[9] F Dahmani, Combination of convergence groups, Geom. Topol. 7 (2003) 933-963 MR2026551

[10] B Farb, Relatively hyperbolic groups, Geom. Funct. Anal. 8 (1998) 810-840 MR1650094

[11] F Gautero, Geodesics in trees of hyperbolic and relatively hyperbolic groups arXiv: 0710.4079

[12] E Ghys, P de la Harpe, editor, Sur les groupes hyperboliques d'après Mikhael Gromov, Progress in Math. 83, Birkhäuser, Boston (1990) MR1086648Papers from the Swiss Seminar on Hyperbolic Groups held in Bern, 1988

[13] R Gitik, M Mitra, E Rips, M Sageev, Widths of subgroups, Trans. Amer. Math. Soc. 350 (1998) 321-329 MR1389776

[14] M Gromov, Hyperbolic groups, from: "Essays in group theory", Math. Sci. Res. Inst. Publ. 8, Springer, New York (1987) 75-263 MR919829

[15] G C Hruska, D T Wise, Packing subgroups in relatively hyperbolic groups arXiv: math.GR/0609369

[16] M Kapovich, Hyperbolic manifolds and discrete groups, Progress in Math. 183, Birkhäuser, Boston (2001) MR1792613

[17] E Klarreich, Semiconjugacies between Kleinian group actions on the Riemann sphere, Amer. J. Math. 121 (1999) 1031-1078 MR1713300 
[18] M Mitra, Maps on boundaries of hyperbolic metric spaces, PhD thesis, UC Berkeley (1997)

[19] M Mitra, Cannon-Thurston maps for trees of hyperbolic metric spaces, J. Differential Geom. 48 (1998) 135-164 MR1622603

[20] M Mitra, Height in splittings of hyperbolic groups, Proc. Indian Acad. Sci. Math. Sci. 114 (2004) 39-54 MR2040599

[21] M Mj, Cannon-Thurston maps for pared manifolds of bounded geometry arXiv: math.GT/0503581

[22] M Mj, Cannon-Thurston maps for surface groups I: amalgamation geometry and split geometry arXiv:math.GT/0512539

[23] M Mj, Cannon-Thurston maps, i-bounded geometry and a theorem of McMullen arXiv:math.GT/0511041

[24] M Mj, A Pal, Relative hyperbolicity, trees of spaces and Cannon-Thurston maps arXiv:0708.3578

[25] L Mosher, Hyperbolic extensions of groups, J. Pure Appl. Algebra 110 (1996) 305-314 MR1393118

[26] L Mosher, A hyperbolic-by-hyperbolic hyperbolic group, Proc. Amer. Math. Soc. 125 (1997) 3447-3455 MR1443845

[27] A Pal, Cannon-Thurston maps and relative hyperbolicity, $\mathrm{PhD}$ thesis, Indian Statistical Institute, Calcutta (expected 2009)

[28] Z Sela, Diophantine geometry over groups. I. Makanin-Razborov diagrams, Publ. Math. Inst. Hautes Études Sci. (2001) 31-105 MR1863735

[29] G A Swarup, Proof of a weak hyperbolization theorem, Q. J. Math. 51 (2000) 529-533 MR1806457

RKM Vivekananda University

Belur Math, WB-711 202, India

University of Melbourne

Victoria 3010, Australia

brmahan@gmail.com, lawrencereeves@gmail.com

Proposed: Benson Farb

Received: 30 March 2007

Seconded: Walter Neumann, Dave Gabai

Revised: 10 April 2008 